\title{
An Intraarticular Osteoid Osteoma: A Case Report \& Review of Literature
}

\author{
Pant AR ${ }^{1}$, Gupta MK ${ }^{1}$, Santhalia PK ${ }^{1}$, Ahmad K ${ }^{1}$, Kalawar RPS ${ }^{2}$, Rauniyar RK ${ }^{1}$ \\ ${ }^{1}$ Department of Radiodiagnosis and Imaging, ${ }^{2}$ Department of Orthopedics, B.P. Koirala \\ Institute of Health Sciences (BPKIHS), Dharan, Nepal
}

\begin{abstract}
Osteoid osteoma (OO) is one of the common benign bone tumors but an uncommon cause of musculoskeletal pain. Its diagnosis is usually not difficult in classic clinical setup and in typical location in diaphyseal region. However, the diagnosis of juxta or intraarticular osteoid osteoma (IAOO) is challenging because of atypical clinical presentation responsible for delay in diagnosis and treatment. We report a rare case of IAOO as a cause of chronic shoulder pain to make clinician aware to help in its early diagnosis and management. A 28-year-old woman presented with chronic debilitating right shoulder pain. The diagnosis was established on CTscan after 2 years of onset of symptoms because of atypical clinical presentation as a chronic monoarthritis of the shoulder. CTscan demonstrated radiolucent nidus with central calcification with areas of surrounding sclerosis. The tumor was excised surgically and histopathologic examination confirmed the diagnosis of osteoid osteoma. So, in the scenario of an unexplained chronic monoarthritis, the possibility of intraarticular osteoid osteoma should also be kept in mind. CT-scan remains the investigation of choice for demonstrating the nidus and surgical exicision relieves the symptoms.
\end{abstract}

Keywords: CT scan, Intrarticular osteoid osteoma, Shoulder pain, Tumor nidus

\section{Introduction}

Osteoid osteoma (OO) is a small and painful benign osteoblastic tumour described for the first time as a separate entity by Jaffe in 1935. ${ }^{1}$ It is located preferentially in the shaft of long bones near the metaphyseal junctions, with a predilection for the lower

Correspondence to: Dr. Ashok Raj Pant, Senior Resident, Department of Radiodiagnosis and Imaging, B.P. Koirala Institute of Health Sciences, Dharan, Nepal.

E-mail: drpantash337@gmail.com limbs. ${ }^{2}$ Juxta and intraarticular OOs are rare and they may be found in any joint, although hip, elbow and talus are the most common location reported in the literature. ${ }^{3}$ They account for approximately $13 \%$ of all osteoid osteomas.

For orthopedicians, most of OO no longer presents a diagnostic problem, because of the characteristic clinical, radiologic and scintigraphic findings. The classic symptoms, however, may not always be present, or they can be misleading, especially when the tumor is located intraarticularly. Because of the unusual clinical and 


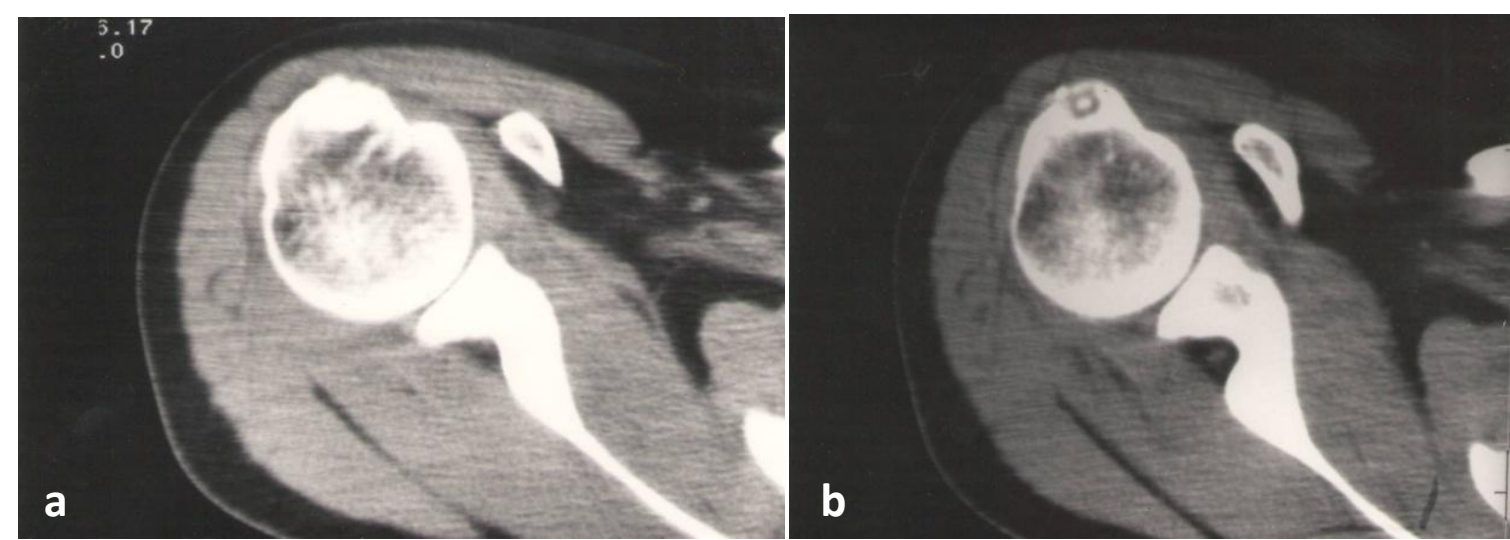

Fig. 1: Axial NCCT soft tissue window (a) and bone window (b) shows a well defined lytic lesion of size $1 \mathrm{~cm}$ with central calcification (s/o calcification in the nidus) with minimal surrounding sclerosis in the anterior cortex of the humeral head.

radiological features of this entity, intraarticular osteoid osteoma (IAOO) is described as a diagnostic challenge. ${ }^{4}$

\section{Case Report}

A 28-year-old woman had complaint of progressive nocturnal debilitating right shoulder pain for approximately 2 years. She visited local practioner, who prescribed non steroidal anti-inflammatory drugs (NSAIDS). Although, she was relieved a bit with these medications, she used to have pain again after discontinuation of the treatment.

Then, she was referred to our hospital where thorough clinical examination was done. Shoulder evaluation revealed normal range of motion and neurovascular findings. Plain film radiograph of the right shoulder showed minimal sclerosis in the right humeral head. Non contrast computed tomography (NCCT) of the right shoulder (Fig. 1) revealed a small well defined lytic lesion with central calcification (s/o nidus) of size $\sim 1 \mathrm{~cm}$ in the anterior cortex of the humeral head with surrounding sclerosis suggesting intraarticular osteoid osteoma. Complete surgical excision of the tumor nidus was performed and the specimen was sent for histopathologic examination which confirmed the diagnosis of intraarticular osteoid osteoma. Post operative course was uneventful with good clinical outcome.

\section{Discussion}

Osteoid osteoma is a benign bone-forming tumor that involves cortical or cancellous bone. The lesion is usually less than $1.5 \mathrm{~cm}$ in size. ${ }^{4}$ The lesion may occur in any bone, although there is a predilection for the lower extremity, with $50 \%$ or more of lesions occurring in the femur and tibia. ${ }^{2}$

Pain is the most prominent symptom of the lesion. It is usually described as mild and intermittent at first, later becoming more constant and severe. Before the lesion is apparent radiologically, in some cases, intense pain may be present. Pain is frequently worse at night and may awaken the patient from sleep and often is relieved by aspirin or NSAIDs. The high levels of prostaglandins within the nidus and the unmyelinated nerve fibers identified in the fibrous zone around the central nidus or in the nidus itself are believed to be the cause 
of pain. ${ }^{5}$ This is the reason why NSAID's can dramatically relieve the pain.

The imaging modality of choice for identifying the nidus of an $\mathrm{OO}$ is the CT. A CT scan usually gives a sharp contrast between the nidus and the surrounding normal trabecular bone even in the absence of a sclerotic rim. Rarely, CT results may be falsely negative. In our case there was a lucent nidus with central calcification with mild surrounding sclerosis. MRI is less useful than CT, and may miss small lesions, or show nonspecific changes. Further, soft tissue and bone edema around the nidus can be confusing and can suggest an aggressive or malignant process.

Initial treatment of $\mathrm{OO}$ involves the use of NSAIDs or aspirin. ${ }^{6}$ Complete surgical excision of the $\mathrm{OO}$ nidus is curative and brings immediate and dramatic relief of symptoms, and is still the preferred treatment method. ${ }^{7}$ Presently, alternative, less invasive methods, such as the percutaneous CT guided radiofrequency ablation, are gaining popularity. In our case complete surgical excision of tumor nidus was performed.

In case of intraarticular location the clinical signs differ significantly from the well known classical symptoms of extraarticular lesion. ${ }^{8}$ The patient of IAOO may present with the complain of articular pain, joint tenderness \& effusion, soft-tissue swelling, stiffness \& decreased range of movement. Various entities that should be taken under consideration in differential diagnosis include synovitis, early osteoarthritis, monoarticular rheumatoid arthritis, inflammatory arthritis, tuberculous arthritis, septic arthritis, Brodie's abscess and intracortical chondroblastoma. ${ }^{9}$
IAOO are most commonly encountered in the hip. Small series have been reported in other joints such as the elbow, the talus, the carpal joints, the spine, shoulder joint and the foot joints ${ }^{3,4}$, however the majority of these IAOO have been described as case reports. In our case, patient presented with monoarticular pain in shoulder joint.

In contrast to the more common cortical OO, IAOO present a diagnostic challenge to the orthopaedic surgeon. The intensity of the sclerosis around the nidus depends on the anatomic location of the lesion, so it is intensive in the diaphysis of a long bone and only mild in the substance of an epimetaphyseal trabecular bone.

Therefore, IAOO in conventional radiographs presents little or no reactive sclerosis. ${ }^{10}$ The nidus is detected in fewer than $50 \%$ of cases when it is below $3 \mathrm{~mm}$ in size. Because of the functional differences between intra- and extracapsular periosteum there is a lack of extensive reactive sclerosis. The less pronounced sclerosis around an intraarticularly located nidus may also explain why bone scan shows increased uptake of tracer in the region of joints but it is nonspecific and often too diffuse for visualization of a nidus. ${ }^{11}$ So, when used alone, it is less helpful in the differential diagnosis.

Hence, because of the nonspecific symptoms and the lack of characteristic radiological features of the IAOO, there is frequent delay in diagnosis and treatment. Delay up to 2.53.5 years in the diagnosis and treatment are reported. In our case, the diagnosis was established after 2 years of onset of symptoms. 


\section{Conclusion}

This case demonstrates that intraarticular osteoid osteoma may present with clinical features that may mimic other common musculoskeletal conditions of the shoulder. Information from the patient history and diagnostic imaging are important for diagnosis and appropriate management.

\section{References}

1. Jaffe HL. "'Osteoid osteoma', A benign osteoblastic tumour composed of osteoid and atypical bone," Archives of Surgery 1935;31:709-728.

2. Sim FH, Dahlin DC, Beabout JW. "Osteoid osteoma: diagnostic problems," Journal of Bone and Joint Surgery. American 1975;57(2):154-159.

3. Cassar-Pullicino VN, McCall IW, Wan S. "Intraarticular osteoid osteoma," Clinical Radiology 1992;45(3):153-160.

4. Greco F, Tamburrelli F, Ciabattoni G. Prostaglandins in osteoid osteoma. Int Orthop.1991;15(1):35-9

5. Resnick D, Kyriakos M, Greenway G. Tumor and tumor - like lesions of bone: imaging of specific lesions. In Diagnosis of bone and joints disorders. Edited by Resnick D, Niwayama G. Philadelphia: Saunders; 1988;4(2):3616-3888.
6. Efstathopoulos N, Sapkas G, Xypnitos FN, Lazarettos I, Korres D, Nikolaou VS. Recurrent intra-articular osteoid osteoma of the hip after radiofrequency ablation: a case report and review of literature. Cases J 2009;2:6439

7. Szendroi M, Kollo K, Antal I, Lakatos J, Szoke G. Intraarticular osteoid osteoma: clinical features, imaging results, and comparison with extra articular localization. J Rheumatol 2004,31:957964.

8. Kumar SJ, Harcke HT, MacEwen GD, Ger E. Osteoid osteoma of the proximal femur: new techniques in diagnosis and treatment. J Pediatr Orthop 1984,4:669672.

9. Moser RP Jr, Kransdorf MJ, Brower AC, Hudson T, Aoki J, Berrey BH. Sweet : Osteoid osteoma of the elbow. A review of six cases. Skeletal Radiol 1990,19:181-186.

10. Ishida T, Goto T, Motoi N, Mukai K. Intracortical chondroblastoma mimicking intra-articular osteoid osteoma. Skeletal Radiol 2002,31:603-607.

11. Norman A. Persistence or recurrence of pain: a sign of surgical failure is osteoidosteoma. Clin Orthop Relat Res 1978,130:263-266. 